Irish Math. Soc. Bulletin

Number 79, Summer 2017, 92-93

ISSN 0791-5578

\title{
Elena Shchepakina, Vladimir Sobolev and Michael P. Mortell: Singular Perturbations. Introduction to System Order Reduction Methods with Applications, Springer, 2014.
}

ISBN:978-3-319-09569-1, USD 59.99, $212 \mathrm{pp}$.

\author{
REVIEWED BY DMITRII RACHINSKII
}

This book will appeal to undergraduate and graduate students, and researchers in the area of applications of singular perturbations in various fields such as chemical kinetics, combustion, control theory and nonlinear dynamics. It combines analytic singular perturbation methods with the geometric approach based on analysis on integral manifolds. The authors are known experts in this field. The reduction to a low dimensional slow integral manifold underpins the order reduction technique presented in the book.

The book is specially constructed to allow a non-expert to read it from beginning to end. In particular, it could serve as the basis for an excellent graduate course on singular perturbations. It begins very simply and is self-contained to such an extent that it is accessible to upper class undergraduate student in mathematics or physics. New topics are introduced by a smooth transition from previous topics. The presentation proceeds by dealing with progressively more difficult problems, where the theory and the solution techniques are laid out. These techniques are illustrated with a large number of examples drawn from widely diverse areas including reaction kinetics of organometallic compounds, combustion problems, population models, control of gyroscopic and robotic systems, and laser dynamics. The examples are completely worked out and become more sophisticated and challenging as the text moves on, until finally they are at the research level where many of the details of calculations in published papers are given. In the earlier chapters there are many simpler examples enabling the reader to get a good grasp of underlying ideas. This exposition style is comparable to that of another major book in the subject area by P. Kokotovic, H. K. Khalil and J. O'Reilly [1].

Received on 9-3-2017. 
In each chapter, the basics are introduced, some theory is presented in a relatively simple way, and then there are examples showing how to apply the method.

Chapters 1 and 2 present an introduction to perturbation methods and integral manifolds.

Chapter 3 gives an overview of examples of increasing complexity with up to two slow variables and up to two fast variables.

Chapter 4 presents a method of finding integral manifolds in parametric and implicit form.

Chapter 5 lays out a technique of scaling transformations and gauge functions, which regularizes singular singularly perturbed problems in order to approximate integral manifolds.

In Chapter 6, the techniques developed so far are applied to order reduction problems.

The full strength of the methodology presented in the book is seen in Chapters 7 and 8 in which the usual hypotheses of integral manifold theory such as the conditions of Tikhonov's theorem are violated. These chapters should be of particular interest to those researchers who need perturbation methods for solving systems of strongly nonlinear equations. The exposition includes mathematical treatment of fascinating slow-fast phenomena represented by cascades of canard solutions and integral surfaces with variable stability called black swans.

\section{REFERENCES}

[1] P. Kokotovic, H. K. Khalil and J. O'Reilly. Singular Perturbation Methods in Control: Analysis and Design. Academic Press, 1986.

Dmitrii Rachinskii works in areas of nonlinear analysis, dynamical systems and mathematical modelling. He recieved $\mathrm{PhD}$ in 1997 from Moscow Institute of Physics and Technology (advisor Mark A. Krasnosel'skii). Before taking the position of professor of Mathematics at UT Dallas, he worked at Russian Academy of Sciences, Weierstrass Institute in Berlin and University College Cork.

Department of Mathematical Sciences, The University of Texas AT DALlas

E-mail address: dmitry.rachinsky@utdallas.edu 